\title{
What Can Trigger Chinese Female Self-Objectification: Revealing Clothes, Tight-Fitting Clothes, or Both?
}

\author{
Yixin Jiang \\ College of Resources and Environment, Southwest University, Chongqing, China \\ Email:18716408392@163.com
}

How to cite this paper: Jiang, Y.X. (2018) What Can Trigger Chinese Female SelfObjectification: Revealing Clothes, TightFitting Clothes, or Both? Open Journal of Social Sciences, 6, 208-223. https://doi.org/10.4236/jss.2018.64018

Received: March 21, 2018

Accepted: April 25, 2018

Published: April 28, 2018

Copyright $\odot 2018$ by author and Scientific Research Publishing Inc. This work is licensed under the Creative Commons Attribution International License (CC BY 4.0).

http://creativecommons.org/licenses/by/4.0/

\section{(c) (i) Open Access}

\begin{abstract}
We aimed to assess if type of clothing caused self-objectification. This study was based on the framework of Objectification Theory. 120 Chinese female undergraduates aged 17 - 25 years were divided into 4 groups: revealing tight-fitting clothes, revealing loose clothes, full tight-fitting clothes, and full loose clothes. They were asked to wear clothes of the most suitable size in two settings, a private setting and a public setting. In both settings, we measured state self-objectification before the participants changed into their original clothes. It was indicated that wearing both revealing and tight-fitting clothes could produce female state self-objectification in the Chinese sociocultural environment. In addition, the public setting led to greater state self-objectification than the private setting. We demonstrate that clothing type is an important contributor to self-objectification of contemporary young women and extend the scope and application of Objectification Theory.
\end{abstract}

\section{Keywords}

Self-Objectification, Clothes, Objectification Theory, Chinese Female

\section{Introduction}

Fredrickson and Roberts [1] proposed that the pervasiveness of sexual objectification in contemporary western societies (e.g., the representation of women in the visual mass media) gradually socialized women and girls to adopt an observer's perspective of their physical self. That is, they come to treat themselves in objectified terms, as an object to be looked at and evaluated based on their appearance. This phenomenon is called "self-objectification" and is characterized by habitual body surveillance [2] or body monitoring. Self-objectified women 
were more concerned about external physical attributes such as "What am I like?" rather than internal ones such as "What can I do?" and "How do I feel?" [3]. Self-objectification can be viewed as a trait that is relatively stable across time; however, self-objectification could also be context dependent and triggered or magnified in certain situations [1]. As the Objectification Theory [1] predicted, the self-objectification could develop a cluster of negative psychological consequences including body shame, appearance anxiety, reduced "flow" states, and decreased awareness of internal states such as hunger and sexual arousal. These intermediary psychological factors, in turn, can promote eating disorders, depression, and sexual dysfunctions. Therefore, in order to protect women from the above negative experiences and mental illness, it is important to explore the contextual determinants of self-objectification.

Every day women enter in and out of multiple contexts, some of which protect them from objectification and others do not. Based on a review of previous research, Fuller-Tyszkiewicz, Reynard, Skouteris and McCabe [4] found that a variety of contextual cues such as exposure to beauty magazines, visual inspection by strangers, receiving a body-related comment, being deprived of intimacy in social interactions, being in situations where one's body is exposed, and presence of a stranger or romantic partner can elicit and promote female self-objectification. Besides, type of clothing may be a relatively important contributor to female self-objectification [5]. In daily life, women spend the majority of their waking hours in clothing, particularly in the public domain, so it is important to elucidate the role of clothing type in female self-objectification.

Fredrickson, Roberts, Noll, Quinn and Twenge [3] found that trying on a swimsuit compared to a bulky sweater resulted in increased self-objectification in their United States college students' sample. A few years later, Hebl, King and Lin [6] using the same procedure studied ethnic differences in self-objectification in a sample of Caucasian, African American, Hispanic, and Asian American undergraduate women, and concurred with the previous results held for the Caucasian sample [7]. In a sample consisting of American undergraduates, Quinn, Callen and Cathey [8] found that other than having higher self-objectification, girls wearing swimsuits listed more body-related thoughts during a free response task given after they had re-dressed, which demonstrated the lingering effect of clothing situations on state self-objectification. Tiggemann et al. [5] in their study on Australian female undergraduate students asked the participants to imagine themselves in bathing suits or a sweater with jeans on the beach or in the dressing room. Results revealed that the scenarios involving bathing suits led to greater state self-objectification. Although previous research elucidated the role of revealing and tight-fitting clothing on female self-objectification using an experimental approach, they could not ascertain whether it is revealing clothes or tight-fitting clothes which really triggered female self-objectification. Namely, research on the effect of clothing type on women is inconclusive.

In a cross-sectional study that investigated the role of wearing revealing 
clothes, American female collegiate cheerleaders reported that they experienced most body dissatisfaction when wearing most revealing uniforms compared to wearing daily clothing and full uniforms [9]. Similarly, in an American female collegiate dancers' sample and an American female collegiate equestrian athletes' sample, Torres-McGehee et al. [9] [10] reported the same results. Although the above research did not explicitly address the role of self-objectification, the theoretical framework provided by Objectification Theory [1] might be particularly useful. In contrast to these results, in an Australian sample of female undergraduates aged 18 - 46 years, Fuller-Tyszkiewicz et al. [4] using a discrete choice conjoint design found that being fully clothed was most likely relative to the other two clothing levels (moderate and minimal) to prompt feelings of self-consciousness for participants, followed by moderately clothed, and then minimally clothed, situations. They reasoned that another aspect (such as the tight-fitting nature of clothing) might be more important for eliciting appearance-related self-consciousness. Accordingly, some researchers explored the effect of tight-fitting clothing on female self-objectification. In a qualitative study investigating women's experiences of dress fit and body image in a UK sample of 20 women aged 18 - 45 years [11], all women reported some self-surveillance in relation to clothes fit, and tightness of clothes resulted in decreased body confidence even in women who were generally body-confident. Similarly, in an Australian sample involving participants and instructors at a fitness center, Prichard and Tiggemann [12] found that the wearing of tight-fitting gym clothing was positively associated with increased self-objectification and self-surveillance, whereas wearing looser clothing was positively related to lower self-objectification and self-surveillance.

The results from these correlational studies and even interview surveys could not prove causal relationships between self-objectification and revealing or tight-fitting clothing. Besides, there was lack of consensus among previous studies regarding effect of revealing clothing on self-objectification. From the above literature review, it is unclear whether it is revealing clothes or tight-fitting clothes which really trigger female self-objectification. Therefore, we aimed to determine if revealing clothes, tight-fitting clothes and revealing tight-fitting clothes resulted in self-objectification. We conducted the study based on the framework of Objectification Theory.

Objectification theory and the concept of self-objectification were originally investigated with predominantly White college women and a few women in other ethnicities. Hebl et al. [6] found that donning a swimsuit compared to a bulky sweater resulted in increased self-objectification in the Asian American college students' sample, confirming that objectification theory could apply to Asian Americans. However, in the literature, few researchers have explored effect of clothing type on self-objectification of local Asian women including Chinese women. In a sample of Chinese college students, Sun et al. [13] found that Chinese women wearing a swimsuit received more objectifying gaze from both women and men than ones clothed in a long sleeved t-shirt and dark pants. Ob- 
jectifying gaze from a stranger is able to improve female self-monitoring [14], so it is probable that Chinese women wearing a swimsuit can experience higher level of self-objectification than who clothed in a long sleeved t-shirt. The current study planed to probe effect of clothing type on self-objectification in a sample of Chinese women.

Similar to the role of clothing type, the presence of a stranger was more likely than the presence of friends to elicit female self-objectification [4]. Prichard and Tiggemann [12] found that the time spent exercising within fitness centers where there was presence of some strangers was positively associated with level of female self-objectification in an Australian fitness center. In view of previous researchers exploring effect of strangers' presence on female self-objectification employed correlational approach, we aimed to investigate, using an experimental approach, whether public setting where there were two strangers can trigger higher level of self-objectification than private setting where there was no one else.

The central purpose of the study was to elucidate the role of wearing revealing clothing and tight-fitting clothing on self-objectification within the framework of Objectification Theory. We investigated the effect of wearing revealing clothing and full clothing across wearing tight-fitting clothing and loose clothing on Chinese female undergraduates in two different settings. Consequently, there were four clothing conditions $(2 \times 2)$ in each of the two settings, which were revealing tight-fitting clothing, revealing loose clothing, full tight-fitting clothing, and full loose clothing. One was simulative dressing room used as private setting, and the other was designed as a public study lounge. The following hypotheses were formulated:

Hypothesis 1a. Wearing revealing clothes will trigger a significantly higher level of state self-objectification than wearing full clothes.

Hypothesis 1b. Wearing tight-fitting clothes compared to wearing loose clothes will elicit a significantly higher level of state self-objectification.

Hypothesis 1c. Wearing revealing tight-fitting clothes will trigger the greatest state self-objectification among four clothing conditions.

Hypothesis 2. There will be a significant interaction effect between the setting condition and the clothes condition on state self-objectification, whereby the difference between a private setting and a public setting will be greater in the revealing tight-fitting clothing, revealing loose clothing, and full tight-fitting clothing groups than in the full loose clothing group.

\section{Method}

\subsection{Participants}

Participants were 120 female undergraduate students from Southwest University (SWU) in Chongqing, China. They were all indigenous Chinese whose age ranged from 18 to 25 years, with a mean age of 20.68 years $(S D=1.78)$. Body Mass Index (BMI), a measure of body fat relative to weight, was calculated using 
the formula $\mathrm{kg} / \mathrm{m}^{2}$ [15]. Participants' BMI ranged from 18.50 to 22.06 with a mean of $19.51(S D=1.50)$, which fell well within the "normal weight" range in China [16].

\subsection{Design}

The study employed a 2 (revealing nature of clothing: revealing, full) $\times 2$ (tight-fitting nature of clothing: tight-fitting, loose) $\times 2$ (setting: private, public) mixed design. Dependent variable was state self-objectification.

\subsection{Materials}

\subsubsection{Self-Objectification Questionnaire}

This questionnaire, developed and validated by Noll and Fredrickson [3], was used to assess trait self-objectification. Participants were asked to rank 10 body attributes in terms of importance to their physical self-concept. The list contains five appearance-based attributes (weight, sex appeal, physical attractiveness, firm or sculpted muscles, and measurements), and five competence-based attributes (physical coordination, health, strength, energy level, and physical fitness level). An individual's score is determined by subtracting the sum of the ranks for the appearance attributes from the sum of the ranks for the competence attributes. Potential scores range from -25 to +25 . A higher score reflected a greater reliance on an appearance-based physical self-concept, indicating greater trait self-objectification [3]. As an ipsative measure, it is not possible to calculate internal consistency reliability for the Self-Objectification Questionnaire. However, construct validity has previously been demonstrated by moderate positive correlations with scores on the Appearance Anxiety Questionnaire $(r=0.52, p<$ $0.01)$ and the Body Image Assessment $(r=0.476, p<0.01)$ (Noll \& Fredrickson, 1998). Its test-retest reliability was $r=0.82(p<0.001)$ in this sample.

\subsubsection{Body Surveillance Subscale}

We used a modified version of a subscale of the Objectified Body Consciousness Scale [2] to measure state self-objectification. Following Breines, Crocker, and Garcia [17], the measure was converted into a state version by asking participants to respond to the statements "right now." Exemplar items include "Right now, I am thinking about how I look" and "Right now, I am more concerned with what my body can do than how it looks." This scale consists of 8 items to which participants respond using a 7-point scale ranging from 1 (strongly disagree) to 7 (strongly agree). Responses are summed to produce a score ranging from 8 to 56, with higher scores representing higher levels of state self-objectification. The internal reliability for the scale was acceptable ( $\alpha=0.75$ for private setting and $\alpha=0.80$ for public setting).

\subsubsection{Demographics}

Questionnaire I. Participants were asked to self-report their age, race, height, weight, clothing size (small, medium and large) and how often they shopped for clothes (less than once a month, once a month, twice a month, three times a 
month, four times a month and more than four times a month).

Questionnaire II. Participants were instructed to rate their experience regarding fit extent and "revealingness" extent of clothing using a 5-point scale ranging from 1 (strongly loose or full) to 5 (strongly tight-fitting or revealing). They also rated how did they like the design of clothing and what did they think of the comfort of clothing using a 5-point scale ranging from 1 (I don't like it at all or It's not comfortable at all) to 5 (I like it very much or It's very comfortable).

\subsubsection{Clothing}

There were four suits used in four clothing conditions respectively. All of the clothing were cotton, grey, and of basic style. They were available in small, medium and large sizes. A skintight midriff waistcoat with close-fitting mini shorts was used as revealing tight-fitting clothing. A loose midriff waistcoat with baggy mini shorts represented for revealing loose clothing. A skintight long-sleeved T-shirt with close-fitting trousers was a representation for full tight-fitting clothing. A loose long-sleeved T-shirt with baggy trousers was used as full loose clothing. See Appendix (Figures A1-A4) for pictures of the clothing.

\subsection{Procedure}

Following approval from the Human Research Ethics committee at SWU, participants were recruited via the campus electronic bulletin board system. Subsequently, 157 undergraduate women were engaged in the initial screening. They completed the Self-Objectification Questionnaire and Demographics Questionnaire I. To ensure that every participant could find the suitable garment size from what we offered, we asked 120 participants who were within the range of normal weight (BMI: 18.5 - 22.79) to take part in a follow-up study. All 120 participants were divided into four groups randomly and each group consisting of 30 participants tried on one of four suits in each setting. They were tested separately. All instructions and questionnaires were presented in Chinese.

\subsubsection{Private Setting}

It was conducted about one week after initial screen, in order to introduce a lag between them. Participants first signed an informed consent. Then they were informed that the study concerned "emotions and consumer decisions" and they would be evaluating everyday items. Participants first evaluated a unisex scent in order to bolster this cover story [6] [7] [8].

The next task was to try on and evaluate a suit of clothing in a completely private dressing room, which had an appropriate temperature setting, a full-length mirror, and a desk. The investigator was not physically present in the setting and participants received the instructions for the tasks over headphones. Participants were asked to select the garment that most closely corresponded to their size and to try it on. Instructions then asked the participants to look at themselves in the mirror for three minutes and then complete a set of questionnaires including 
Body Surveillance Subscale and some other evaluations for the clothing to support the story. In the end, participants went out of the dressing room in their own clothing.

\subsubsection{Public Setting}

It was conducted about three weeks after private setting. The procedure was the same as one in the private setting until participants completed the task of looking at themselves in the mirror for three minutes. Later, the participants wearing the same clothes as they did in private setting, went out of the dressing room and into the next-door public study lounge of appropriate temperature where there were one female student and one male student reading. The two students were experimental assistants. Participants needed to complete the same questionnaires as that in private setting and then changed into their original clothing in dressing room. Next, participants went out of the dressing room in their own clothing and completed the demographics questionnaire II. At last, the participants were asked to guess the main research purpose or hypotheses and none of them guessed it correctly. Following debriefing, participants were paid 15 Yuan as compensation for their time.

\section{Results}

\subsection{Preliminary Analyses}

\subsubsection{Characteristics of the Sample}

On average women in the sample reported shopping for clothes once or twice a month. Thirty participants' usual clothes size was small, sixty-six of them wore medium sized and twenty-four of them wore large sized clothes. Size of the outfits picked in the experiment matched the clothing size of most participants.

\subsubsection{Validity Check on Clothing}

In an effort to examine the validity of experiment's manipulation of clothing, the study performed a between-subjects 2 (revealing nature of clothing: revealing, full) $\times 2$ (tight-fitting nature of clothing: tight-fitting, loose) analysis of variance (ANOVA) for participants' experience to fit extent and revealingness extent of clothing. Table 1 provides means and standard deviations for the two measures, as well as for the four clothing conditions. There was a significant main effect for revealing nature of clothing with experience to revealingness extent as the dependent variable $\left(M_{\text {revealing,clothes }}=3.23, S D=0.81, M_{\text {full,clothes }}=1.35, S D=0.48\right)$, $F(1,116)=249.03, p<0.0001, \eta_{p}^{2}=0.68$, but not for tight-fitting nature of clothing $\left(M_{\text {tight-fitting,clothes }}=2.43, S D=1.25, M_{\text {loose,clothes }}=2.15, S D=1.04\right)$. There was also no significant the interaction effect $\left(p_{\mathrm{s}}>0.05\right)$. There was a significant main effect for tight-fitting nature of clothing with scores of experience to fit extent as the dependent variable $\left(M_{\text {tight-fitting,clothes }}=3.73, S D=0.66, M_{\text {loose,clothes }}=\right.$ 2.15, $S D=0.66), F(1,116)=185.75, p<0.0001, \quad \eta_{p}^{2}=0.62$ but not for revealing nature of clothing $\left(M_{\text {revealing,clothes }}=2.78, S D=0.90, M_{\text {full,clothes }}=3.10, S D=1.13\right)$, 
Table 1. Mean scores $(S D)$ for measured extraneous variables.

\begin{tabular}{ccccc}
\hline & \multicolumn{2}{c}{ Revealing clothes } & \multicolumn{2}{c}{ Full clothes } \\
\cline { 2 - 5 } & $\begin{array}{c}\text { Tight-fitting } \\
\text { clothes } \\
(N=30)\end{array}$ & $\begin{array}{c}\text { Loose } \\
\text { clothes } \\
(N=30)\end{array}$ & $\begin{array}{c}\text { Tight-fitting } \\
\text { clothes } \\
(N=30)\end{array}$ & $\begin{array}{c}\text { Loose clothes } \\
(N=30)\end{array}$ \\
\hline $\begin{array}{c}\text { Experience to } \\
\text { revealingness extent } \\
\text { Experience to } \\
\text { fit extent }\end{array}$ & $3.43(0.94)$ & $3.03(0.61)$ & $1.43(0.50)$ & $1.27(0.45)$ \\
$\begin{array}{c}\text { Trait self-objectification } \\
\text { BMI }\end{array}$ & $-4.47(0.57)$ & $2.10(0.61)$ & $4.00(0.64)$ & $2.20(0.71)$ \\
$\begin{array}{c}\text { "How did they like the } \\
\text { design of clothing" }\end{array}$ & $19.20(1.42)$ & $19.64(1.51)$ & $19.38(1.77)$ & $19.71(1.35)$ \\
$\begin{array}{c}\text { "How did they think the } \\
\text { comfort of clothing" }\end{array}$ & $2.70(0.92)$ & $2.50(0.78)$ & $2.43(0.86)$ & $2.17(0.95)$ \\
\hline
\end{tabular}

or the interaction effect $\left(p_{\mathrm{s}}>0.05\right)$. The results indicates that the fit extent and revealingness extent of clothing that participants experienced concurred with the research predictions of the experiment.

\subsubsection{Group Equivalency Checks}

In order to assess that the groups were equivalent in preference for the design of clothing and comfort of clothing, the study performed a 2 (revealing nature of clothing: revealing, full) $\times 2$ (tight-fitting nature of clothing: tight-fitting, loose) between-subjects analysis of variance (ANOVA). The respective means and standard deviations for each dependent variable are shown in Table 1. Neither preference nor comfort differed significantly between wearing revealing clothing and wearing full clothing (respective $p_{\mathrm{s}}=0.06,0.46$ ) or between wearing tight-fitting clothing and wearing loose clothing (respective $p_{\mathrm{s}}=0.15,0.34$ ). There was also no significant interaction effect (respective $p_{\mathrm{s}}=0.21,0.85$ ), indicating there was no difference among four groups on preference for the design of clothing and comfort of clothing.

The four groups were created using random assignment procedure and was predicted not to differ significantly on trait self-objectification and BMI. A 2 (revealing nature of clothing: revealing, full) $\times 2$ (tight-fitting nature of clothing: tight-fitting, loose) between-subjects analysis of variance (ANOVA) were conducted, with trait self-objectification and BMI as the dependent variable. The respective means for each dependent variable are reported in Table 1. Neither the trait self-objectification nor BMI differed between wearing revealing clothing and wearing full clothing (respective $p_{\mathrm{s}}=0.12,0.66$ ) or between wearing tight-fitting clothing and wearing loose clothing (respective $p_{\mathrm{s}}=0.79,0.17$ ). There was also no significant interaction effect (respective $p_{\mathrm{s}}=0.21,0.85$ ). The results indicated that there was no difference among the four groups on trait self-objectification and BMI. Therefore, trait self-objectification as well as BMI was not considered into following statistics analysis. 


\subsection{Experiment Effect on State Self-Objectification}

\subsubsection{Analyses of Variance}

In order to investigate the effect of experiment effect on state self-objectification, a 2 (revealing nature of clothing: revealing, full) $\times 2$ (tight-fitting nature of clothing: tight-fitting, loose) $\times 2$ (setting: private, public) repeated measure ANOVA was conducted, with state self-objectification as the dependent variable. Table 2 displays the means scores and standard deviations for the eight conditions.

The results indicated a significant main effect of revealing nature of clothing on state self-objectification, $F(1,116)=10.40, p=0.002, \eta_{p}^{2}=0.14$, whereby wearing revealing clothes $(M=38.46, S D=5.77)$ led to significantly higher state self-objectification than wearing full clothes $(M=34.71, S D=6.88)$. Thus, Hypothesis la was supported.

The results revealed there was a significant main effect of tight-fitting nature of clothing on state self-objectification, $F(1,116)=12.94, p<0.001, \eta_{p}^{2}=0.16$, whereby wearing tight-fitting clothes $(M=38.51, S D=6.20)$ led to significantly higher state self-objectification than wearing loose clothes $(M=34.21, S D=$ 6.40). Hence, Hypothesis $1 \mathrm{~b}$ was supported.

Besides, there was a significant main effect for setting, $F(1,116)=12.93, p<$ $0.001, \eta_{p}^{2}=0.16$, whereby the public setting led to higher state self-objectification $(M=38.05, S D=6.26)$ than the private setting $(M=35.12, S D=6.73)$. The results showed there was not any two-factor or three-factor significant interaction $\left(p_{\mathrm{s}}>0.05\right)$. Thus, our study results did not support Hypothesis 2 .

\subsubsection{Multiple Comparison Procedure}

In an effort to rank the state self-objectification of four clothing conditions in each setting, two one-way ANOVA and following post hoc tests (LSD) were conducted, with state self-objectification as the dependent variable. The results showed a significant main effect of clothing type on state self-objectification in the private setting, $F(3,116)=7.16, p<0.001, \eta_{p}^{2}=0.18$, and a significant main

Table 2. Mean scores and standard deviations for State self-objectification by clothing and settings.

\begin{tabular}{ccccc}
\hline & \multicolumn{2}{c}{ Revealing clothing } & \multicolumn{2}{c}{ Full clothing } \\
\cline { 2 - 5 } & $\begin{array}{c}\text { Tight-fitting } \\
\text { clothing } \\
(N=30)\end{array}$ & $\begin{array}{c}\text { Loose clothing } \\
(N=30)\end{array}$ & $\begin{array}{c}\text { Tight-fitting } \\
\text { clothing } \\
(N=30)\end{array}$ & $\begin{array}{c}\text { Loose clothing } \\
(N=30)\end{array}$ \\
\hline \multicolumn{2}{c}{ Private setting } & & & $30.80_{\mathrm{c}}$ \\
$M$ & $38.32_{\mathrm{a}}$ & $36.68_{\mathrm{ab}}$ & $34.68_{\mathrm{b}}$ & $(6.59)$ \\
\hline$(S D)$ & $(5.61)$ & $(6.39)$ & $(5.57)$ & $33.90_{\mathrm{c}}$ \\
\hline \multicolumn{2}{c}{ Public setting } & & & $(6.41)$ \\
\hline
\end{tabular}

Note: Means were ranked by $a, b$, and c ordered by the magnitude of the value $(a>b>c)$. 
effect of clothing type on state self-objectification in the public setting, $F(3,116)=7.13, p<0.001, \eta_{p}^{2}=0.22$. Table 2 presents the means of state self-objectification scores of each clothing condition ranked by a, b, and $c$, and ordered by the magnitude of the value $(a>b>c)$. As can be seen in Table 2, wearing revealing tight-fitting clothing triggered significantly greater state self-objectification than wearing full loose clothing (control condition) in both settings, while it triggered significantly greater state self-objectification than wearing revealing loose clothing only in the private setting and wearing full tight-fitting clothing only in the public setting. So, Hypothesis $1 \mathrm{c}$ received limited support.

\section{Discussion}

Based on the framework of Objectification Theory, we successfully triggered female state self-objectification by daily clothes and simulative settings, creatively compared the effect of wearing revealing clothes and wearing tight-fitting clothes on female self-objectification, and demonstrated cross-cultural consistency of Objectification Theory with the Chinese sample. Therefore, we both extend the scope and application of Objectification Theory and adds to the scant existing literature on clothing.

The major finding was that both wearing revealing clothes and wearing tight-fitting clothes were contextual cues that could result in increased state self-objectification, and wearing tight-fitting revealing clothes could occasionally trigger greater state self-objectification than wearing revealing loose clothes and full tight-fitting clothes. Besides, our results-that wearing revealing clothes led to significantly greater state self-objectification than wearing full clothes-corroborates the argument about the role of wearing revealing clothes on self-objectification [4] [9]. The phenomenon that both wearing tight-fitting clothes and revealing clothes can trigger and increase self-objectification and body surveillance may rely on two pathways. On the one hand, in the contemporary sociocultural milieu, women wearing revealing or tight-fitting clothes are easy to be treated as an object to be looked at and evaluated on the basis of appearance, and can receive more objectifying gaze [18] and body-related comments than women wearing full or loose clothes. Objectifying gaze is able to make women adopt an observer's perspective of their physical self and improve self-monitoring of body [14], while appearance-related commentary have been showed to can elicit self-objectification [19] and even appearance compliment could trigger appearance self-consciousness [20]. On the other hand, both tight-fitting clothes and revealing clothes focus on external appearance in some way, which makes women who have high level of internalization of social beauty ideals [21] and social comparison [22] involuntarily evaluate and monitor their bodies more frequently than usual. Thus it appears that not only wearing revealing clothes but also wearing tight-fitting clothes is a negative and stressful experience for most women, considering that increased state self-objectification is accompanied 
by increased state body shame, body dissatisfaction and negative mood (e.g., [20]).

Moreover, we found that both wearing revealing clothes and wearing tight-fitting clothes could result in increased self-objectification of Chinese women, indicating that female self-objectification also existed in the Chinese sociocultural environment. Nowadays, Chinese young female are more concerned about their external appearance than internal ability. Chinese female undergraduates expressed relatively much concerns about facial appearance and fatness [23]. Chinese young women are often consumers of cosmetic procedures, and increasingly are confronted with eating disorder symptoms [24]. Cultural objectification can lead to self-objectification [1] [25] [26]. Living in an objectifying culture socializes women to objectify themselves, that is, to internalize the culture's feminine ideal of thinness, youthfulness, attractiveness, and physical perfection, and to measure one's worth as an individual against this ideal. Actually, objectifying culture has existed in China very early, for example that foot-binding, that can help women to follow social beauty norm in ancient China, has already begun from the Song Dynasty. Over the past thirty years, as western objectifying culture spread internationally on the premise of reform and opening in China, it is possible that Chinese women have had more and more objectified body consciousness. Thus, there is no doubt that female self-objectification existed in the China, though Gervais et al. [27] opined that sexual objectification could only exist in the Western sociocultural environment and could not exist in other areas.

In this study, the public setting led to greater state self-objectification than the private setting. The results that the presence of strangers could increase self-objectification are consistent with the previous study [4]. It can be interpreted as the phenomenon that the objectifying gaze [14] and objectifying comments [19] from strangers were able to improve female self-objectification. Calogero [28] found that mere anticipating a stranger's gaze enhanced female self-objectification, so authors speculated that anticipating a stranger's appearance-related comments was likely to increase female self-objectification in this research in consideration that two simulate readers did not say any words.

Aside from its contribution to the theory, this research has a number of possible practical implications for women's everyday life. As is known to all, revealing or tight-fitting clothing showing female beautiful body shape let women happy in daily life. On the contrary, clothing that completely accentuate or reveal the real body shape to wearers or others could elicit female self-objectification and could trigger a number of negative experiential consequences in an objectifying culture where most women's body are difficult to attain their object about thin hourglass-shaped body. As Fredrickson and Roberts [1] said "The wearing of baggy clothing may be a strategy used by women to opt out of the objectification limelight." The wearing of slightly full and loose clothing may be used as a deliberate strategy by women to consciously decrease self-monitoring of outward 
appearance, as well as the objectifying gaze and comments from others. In particular, the wearing of such clothes likely removes the constant need to monitor oneself and one's reception. In addition, the wearing of loose or full clothing was a probable method for intervening and treating some mental illness led by habitual self-objectification, such as eating disorders and depression.

Secondly, costume designers should be sensitive to the body concerns many women have and design clothing that can cover their physical flaws and even make them look better. Finally, a number of studies have reported that increased self-objectification accompanied with impaired cognitive function might reduce the work efficiency, which raised concerns about female workplace attire. Of course, office wear makes women look competent and professional [29], but fitted or even a little tight-fitting clothing may elicit and enhance self-objectification reducing work efficiency.

Like many studies, the present research findings have certain limitations. First, lack of a baseline measure of state self-objectification is a limitation. However, there was no difference of trait self-objectification in the four groups, which could indicate there was no significant difference on state self-objectification in the four groups. Second, the use of the same sample of participants in both the public and the private setting likely increased the possibility of practice effects and demand characteristics. Third, the sample was restricted to young female university students and so results may not generalize to other groups of women. Nevertheless, clothing and self-objectification are very important issues for this age group [30]. Fourth, the sample was also restricted to young women of normal weight. Maybe there is a dramatic interaction effect between clothing types and weight groups (e.g. under weight, normal weight, over weight), based on the evidence that women who have higher BMI feel the need to camouflage their bodies with less revealing clothing. Adding a greater range of BMI is a feasible next step [31].

In our future research, we plan to measure the negative and stressful experiences such as body shame and body dissatisfaction following self-objectification. Research on self-objectification suggests that the more women do it, the more affective and cognitive problems can result. Adding measures of them is a feasible next step. In addition, it is also important to test causal and mediational models as has been done with studies in the US. In particular, because self-surveillance has been linked with negative body emotions and disordered eating in many US studies, it would be useful to investigate this link in Chinese women.

Further research also could explore the role of color and pattern of clothing, in determining self-objectification. It was found that not only males but also females judged women wearing red clothes owned higher sexual receptivity than women wearing white [32] [33]. Can sexual objectification of women wearing red clothes, lead to female self-objectification? It was also suggested that the wearing of cross stripe clothes made people look thinner than the wearing of vertical stripe clothes when size and type of clothes were controlled [34]. Can it 
reduce body dissatisfaction caused by self-objectification?

\section{Conclusion}

In conclusion, despite its limitations, the present study has made a start on investigating the roles of clothes type (revealing clothes and tight-fitting clothes) in self-objectification and a start on exploring self-objectification in a Chinese female sample. Overall, the results confirmed that tight-fitting clothes, revealing clothes and tight-fitting revealing clothes trigger self-objectification of young women in the Chinese sociocultural environment.

\section{References}

[1] Fredrickson, B.L. and Roberts, T.A. (1997) Objectification Theory: Toward Understanding Women's Lived Experiences and Mental Health Risks. Psychology of Women Quarterly, 21, 173-206. https://doi.org/10.1111/j.1471-6402.1997.tb00108.x

[2] McKinley, N.M. and Hyde, J.S. (1996) The Objectified Body Consciousness Scale Development and Validation. Psychology of Women Quarterly, 20, 181-215. https://doi.org/10.1111/j.1471-6402.1996.tb00467.x

[3] Noll, S.M. and Fredrickson, B.L. (1998) A Mediational Model Linking Self-Objectification, Body Shame, and Disordered Eating. Psychology of Women Quarterly, 22, 623-636. https://doi.org/10.1111/j.1471-6402.1998.tb00181.x

[4] Fuller-Tyszkiewicz, M., Reynard, K., Skouteris, H. and McCabe, M. (2012) An Examination of the Contextual Determinants of Self-Objectification. Psychology of Women Quarterly, 36, 76-87. https://doi.org/10.1177/0361684311426721

[5] Tiggemann, M. and Andrew, R. (2012) Clothes Make A Difference: The Role of Self-Objectification. Sex Roles, 66, 646-654.

https://doi.org/10.1007/s11199-011-0085-3

[6] Hebl, M.R., King, E.B. and Lin, J. (2004) The Swimsuit Becomes Us All: Ethnicity, Gender, and Vulnerability to Self-Objectification. Personality and Social Psychology Bulletin, 30, 1322-1331. https://doi.org/10.1177/0146167204264052

[7] Fredrickson, B.L., Roberts, T.-A., Noll, S.M., Quinn, D.M. and Twenge, J.M. (1998) That Swimsuit Becomes You: Sex Differences in Self-Objectification, Restrained Eating, and Math Performance. Journal of Personality and Social Psychology, 75, 269-284. https://doi.org/10.1037/0022-3514.75.1.269

[8] Quinn, D.M., Kallen, R.W. and Cathey, C. (2006) Body on My Mind: The Lingering Effect of State Self-Objectification. Sex Roles, 55, 869-874. https://doi.org/10.1007/s11199-006-9140-x

[9] Torres-McGehee, T.M., Monsma, E.V., Dompier, T.P. and Washburn, S.A. (2012). Eating Disorder Risk and the Role of Clothing in Collegiate Cheerleaders' Body Images. Journal of Athletic Training, 47, 541-578.

https://doi.org/10.4085/1062-6050-47.5.03

[10] Torres-McGehee, T.M., Green, J.M., Leeper, J.D., Leaver-Dunn, D., Richardson, M. and Bishop, P.A. (2009) Body Image, Anthropometric Measures, and Eating-Disorder Prevalence in Auxiliary Unit Members. Journal of Athletic Training, 44, 418-426. https://doi.org/10.4085/1062-6050-44.4.418

[11] Grogan, S., Gill, S., Brownbridge, K., Kilgariff, S. and Whalley, A. (2013) Dress Fit and Body Image: A Thematic Analysis of Women's Accounts during and after Trying on Dresses. Body Image, 10, 380-388. 
https://doi.org/10.1016/j.bodyim.2013.03.003

[12] Prichard, I. and Tiggemann, M. (2005) Objectification in Fitness Centers: Self-Objectification, Body Dissatisfaction, and Disordered Eating in Aerobic Instructors and Aerobic Participants. Sex Roles, 53, 19-28.

https://doi.org/10.1007/s11199-005-4270-0

[13] Sun, Q., Zheng, L. and Zheng, Y. (2015) The Effect of Sexual Objectification on Perceptions of Intelligence: An Eye Movement Study. Psychological Science, 38, 1187-1193. (In Chinese)

[14] Gervais, S.J., Vescio, T.K. and Allen, J. (2011) When What You See Is What You Get the Consequences of the Objectifying Gaze for Women and Men. Psychology of Women Quarterly, 35, 5-17. https://doi.org/10.1177/0361684310386121

[15] Garrow, J.S. and Webster, J. (1984) Quetelet's Index (w/h2) as a Measure of Fatness. International Journal of Obesity, 9, 147-153.

[16] He, W., Li, Q., Yang, M., Jiao, J., Ma, X., Zhou, Y., et al. (2015) Lower BMI Cutoffs to Define Overweight and Obesity in China. Obesity, 23, 684-691. https://doi.org/10.1002/oby.20995

[17] Breines, J.G., Crocker, J. and Garcia, J.A. (2008) Self-Objectification and Well-Being in Women's Daily Lives. Personality and Social Psychology Bulletin, 34, 583-598. https://doi.org/10.1177/0146167207313727

[18] Holland, E. and Haslam, N. (2013) Worth the Weight. The Objectification of Overweight versus Thin Targets. Psychology of Women Quarterly, 37, 462-468. https://doi.org/10.1177/0361684312474800

[19] Calogero, R.M., Herbozo, S. and Thompson, J.K. (2009) Complimentary Weightism: The Potential Costs of Appearance-Related Commentary for Women's Self-Objectification. Psychology of Women Quarterly, 33, 120-132. https://doi.org/10.1111/j.1471-6402.2008.01479.x

[20] Tiggemann, M. and Boundy, M. (2008) Effect of Environment and Appearance Compliment on College Women's Self-Objectification, Mood, Body Shame, and Cognitive Performance. Psychology of Women Quarterly, 32, 399-405. https://doi.org/10.1111/j.1471-6402.2008.00453.x

[21] Vandenbosch, L. and Eggermont, S. (2012) Understanding Sexual Objectification: A Comprehensive Approach toward Media Exposure and Girls' Internalization of Beauty Ideals, Self-Objectification, and Body Surveillance. Journal of Communication, 62, 869-887. https://doi.org/10.1111/j.1460-2466.2012.01667.x

[22] Lindner, D., Tantleff-Dunn, S. and Jentsch, F. (2012) Social Comparison and the "Circle of Objectification". Sex Roles, 67, 222-235. https://doi.org/10.1007/s11199-012-0175-x

[23] Chen, H., Jackson, T. and Huang, X. (2006) The Negative Physical Self Scale: Initial Development and Validation in Samples of Chinese Adolescents and Young Adults. Body Image, 3, 401-412. https://doi.org/10.1016/j.bodyim.2006.07.005

[24] Chen, H. and Jackson, T. (2008) Prevalence and Sociodemographic Correlates of Eating Disorder Endorsements among Adolescents and Young Adults from China. European Eating Disorders Review, 16, 375-385. https://doi.org/10.1002/erv.837

[25] McKinley, N.M. (1999) Women and Objectified Body Consciousness: Mothers' and Daughters' Body Experience in Cultural, Developmental, and Familial Context. Developmental Psychology, 35, 760-769. https://doi.org/10.1037/0012-1649.35.3.760

[26] McKinley, N.M. (2006) The Developmental and Cultural Contexts of Objectified Body Consciousness: A Longitudinal Analysis of Two Cohorts of Women. Developmental Psychology, 42, 679-687. https://doi.org/10.1037/0012-1649.42.4.679 
[27] Gervais, S.J., Holland, A.M. and Dodd, M.D. (2013) My Eyes Are up Here: The Nature of the Objectifying Gaze toward Women. Sex Roles, 69, 557-570. https://doi.org/10.1007/s11199-013-0316-x

[28] Calogero, R.M. (2004) A Test of Objectification Theory: The Effect of the Male Gaze on Appearance Concerns in College Women. Psychology of Women Quarterly, 28, 16-21. https://doi.org/10.1111/j.1471-6402.2004.00118.x

[29] Gurney, D.J., Howlett, N., Pine, K., Tracey, M. and Moggridge, R. (2017) Dressing up Posture: The Interactive Effects of Posture and Clothing on Competency Judgements. British Journal of Psychology, 108, 436-451. https://doi.org/10.1111/bjop.12209

[30] Lee, Y.A. and Sontag, M.S. (2010) An Assessment of the Proximity of Clothing to Self Scale for Older Persons. International Journal of Consumer Studies, 34, 443-448. https://doi.org/10.1111/j.1470-6431.2010.00874.x

[31] Tiggemann, M. and Lacey, C. (2009) Shopping for Clothes: Body Satisfaction, Appearance Investment, and Functions of Clothing among Female Shoppers. Body Image, 6, 285-291. https://doi.org/10.1016/j.bodyim.2009.07.002

[32] Pazda, A.D., Elliot, A.J. and Greitemeyer, T. (2012) Sexy Red: Perceived Sexual Receptivity Mediates the Red-Attraction Relation in Men Viewing Woman. Journal of Experimental Social Psychology, 48, 787-790. https://doi.org/10.1016/j.jesp.2011.12.009

[33] Pazda, A.D., Prokop, P. and Elliot, A.J. (2014) Red and Romantic Rivalry Viewing Another Woman in Red Increases Perceptions of Sexual Receptivity, Derogation, and Intentions to Mate-Guard. Personality and Social Psychology Bulletin, 40, 1260-1269. https://doi.org/10.1177/0146167214539709

[34] Thompson, P. and Mikellidou, K. (2011) Applying the Helmholtz Illusion to Fashion: Horizontal Stripes Won't Make You Look Fatter. i-Perception, 2, 69-76. https://doi.org/10.1068/i0405 


\section{Appendix}

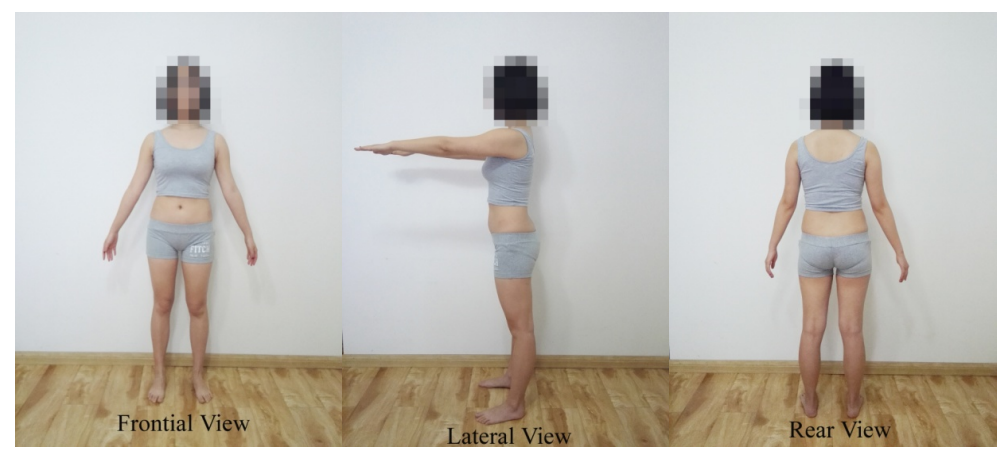

Figure A1. Pictures of revealing tight-fitting clothes.

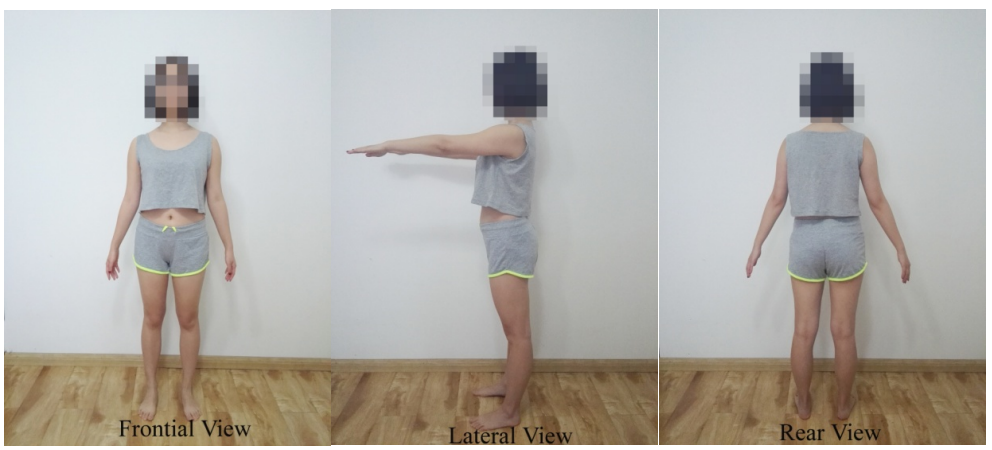

Figure A2. Pictures of revealing loose clothes.

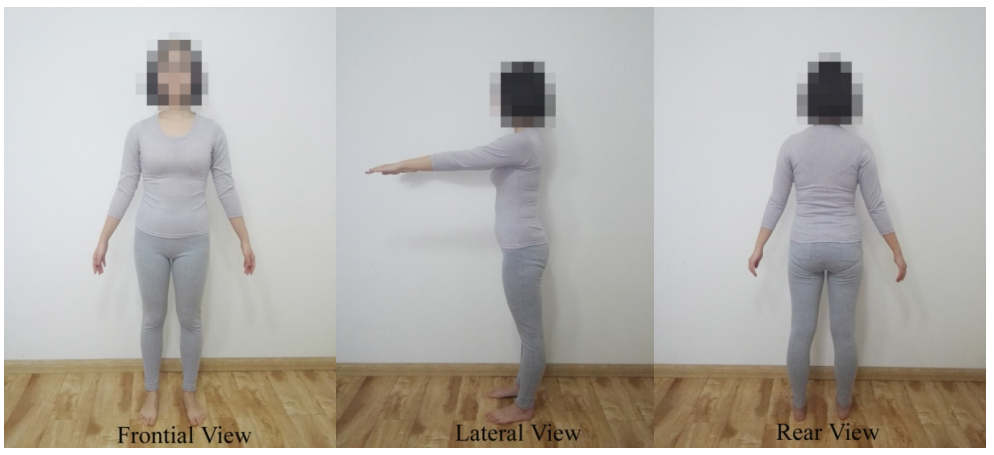

Figure A3. Pictures of full tight-fitting clothes.

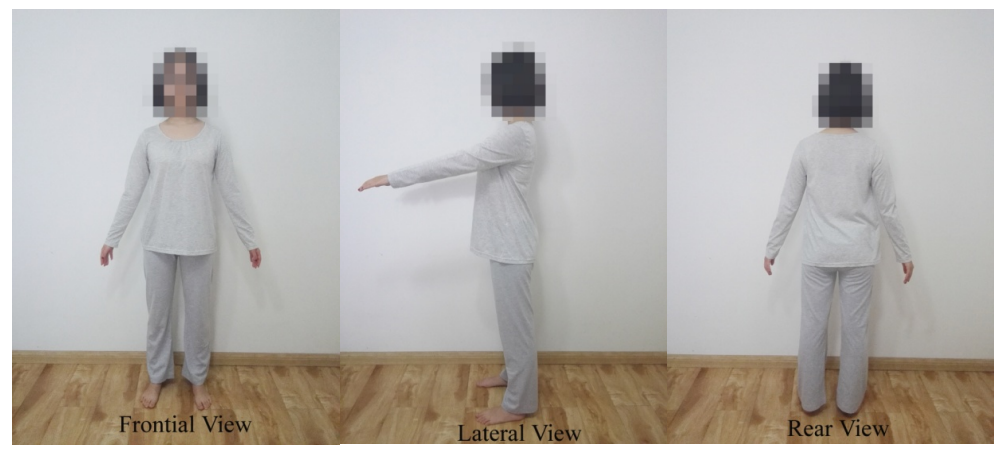

Figure A4. Pictures of full loose clothes. 